\title{
Fluidized bed catalytic chemical vapor deposition synthesis of carbon nanotubes-A review
}

\begin{abstract}
Carbon nanotubes (CNTs) are pure carbon in nanostructures with unique physico-chemical properties. They have brought significant breakthroughs in different fields such as materials, electronic devices, energy storage, separation, sensors, etc. If the CNTs are ever to fulfill their promise as an engineering material, commercial production will be required. Catalytic chemical vapor deposition (CCVD) technique coupled with a suitable reactor is considered as a scalable and relatively low-cost process enabling to produce high yield CNTs. Recent advances on CCVD of CNTs have shown that fluidizedbed reactors have a great potential for commercial production of this valuable material. However, the dominating process parameters which impact upon the CNT nucleation and growth need to be understood to control product morphology, optimize process productivity and scale up the process. This paper discusses a general overview of the key parameters in the CVD formation of CNT. The focus will be then shifted to the fluidized bed reactors as an alternative for commercial production of CNTs.
\end{abstract}

Keyword: Carbon nanotube; Chemical vapor deposition; Fluidized bed; Synthesis; Catalytic 